\title{
Cost-effectiveness of precision cancer medicine-current challenges in the use of next generation sequencing for comprehensive tumour genomic profiling and the role of clinical utility frameworks (Review)
}

\author{
KONSTANTINOS CHRISTOFYLLAKIS ${ }^{1}$, JOERG THOMAS BITTENBRING ${ }^{1}$, \\ LORENZ THURNER $^{1}$, MANFRED AHLGRIMM ${ }^{1}$, STEPHAN STILGENBAUER $^{1-3}$, \\ MORITZ BEWARDER $^{1}$ and DOMINIC KADDU-MULINDWA ${ }^{1}$ \\ ${ }^{1}$ Department of Hematology, Oncology, Clinical Immunology and Rheumatology, \\ Saarland University Medical Center, D-66421 Homburg; ${ }^{2}$ Ulm Comprehensive Cancer Center; \\ ${ }^{3}$ Department of Internal Medicine III, Ulm University Hospital, D-89081 Ulm, Germany
}

Received July 3, 2021; Accepted August 30, 2021

DOI: $10.3892 / \operatorname{mco} .2021 .2453$

\begin{abstract}
Precision cancer medicine (PCM) is an emerging paradigm in oncology, which includes tumour comprehensive genomic profiling (CGP) to enable molecularly guided therapy. However, cost-effectiveness analyses of PCM are faced with several challenges and, thus, its cost-effectiveness remains unclear. Early trials using only molecularly guided therapy were faced with the challenge of providing adequate measures of outcome, which probably explains the modest treatment benefits demonstrated. Endpoints like the progression-free survival (PFS)2/PFS1 ratio may assist in overcoming this issue. Moreover, specific tumour subtypes appear to benefit more from PCM. Costs associated with next-generation sequencing (NGS) for CGP are decreasing, but targeted therapy itself represents a major cost driver. CGP not only enables prediction of response to treatment, but also resistance, and could thus prevent administration of unnecessary (and costly) therapies. In clinical practice, the presence of
\end{abstract}

Correspondence to: Dr Konstantinos Christofyllakis, Department of Hematology, Oncology, Clinical Immunology and Rheumatology, Saarland University Medical Center, 100 Kirrberger Street, D-66421 Homburg, Germany

Email: konstantinos.christofyllakis@uks.eu

Abbreviations: PCM, precision cancer medicine; CGP, comprehensive genomic profiling; NGS, next-generation sequencing; TKI, tyrosine kinase inhibitors; PFS, progression-free survival; NSCLC, non-small cell lung cancer; WES, whole exome sequencing; ESMO, European Society of Medical Oncology; ESCAT, ESMO Scale for Clinical Actionability of Molecular Targets

Key words: precision cancer medicine, cost effectiveness, targeted therapy, next generation sequencing, comprehensive genomic profiling clinical frameworks, such as the Recommendations for the Use of NGS for Patients with Metastatic Cancers from the ESMO Precision Medicine Working Group, and the ESMO Scale for Clinical Actionability of Molecular Targets, are essential in appropriately identifying situations where PCM is clinically meaningful, thereby improving its cost-effectiveness.
Contents
1. Introduction
2. Clinical outcomes
3. Costs
4. Cost effectiveness
5. Clinical utility frameworks
6. Conclusion

\section{Introduction}

Precision cancer medicine (PCM) is an emerging paradigm in cancer treatment striving to tailor anticancer therapy to the individual patient and treatment scenario. Originally, it referred to targeted or biomarker driven therapy (1). Examples of this approach include the use of monoclonal antibodies against cancers expressing a particular antigen/receptor, such as rituximab in CD20-positive B-cell lymphoma, or the use of tyrosine kinase inhibitors (TKIs) targeting a mutated kinase, such as EGFR-TKIs in lung cancer with EGFR driver mutations. With increasing availability and evolution of high-throughput technologies, such as next-generation sequencing (NGS), the concept of PCM has evolved to include comprehensive genetic profiling (CGP) of individual tumours, in order to identify and target alterations that are patient- and tumour-specific (2). This promise of personalized cancer therapy initially appeared alluring; however, the results of prospective trials have been sobering. The increased costs of 
comprehensive molecular tumour profiling for each individual patient and the cost of targeted therapy itself, pose the question of the cost-effectiveness of this paradigm (3). Contrary to the broad application of PCM, streamlining its use in clinical scenarios where it is deemed to be clinically meaningful based on internationally recognised clinical frameworks can be an essential step in increasing its cost-effectiveness.

\section{Clinical outcomes}

The PCM approach has been tested in several prospective trials to date. The SHIVA trial (4) randomized patients to receive either molecularly guided therapy or the physician's choice. Progression-free survival (PFS) was similar in both arms [2.3 months in the experimental group vs. 2.0 months in the control group $(\mathrm{P}=0.41)]$. Trédan et al $(5)$ reported one of the largest series to date, including 2,579 patients with advanced cancers in the ProfiLER trial. Molecularly guided treatment could be recommended for $27 \%$ of the patients, but only $6 \%$ received targeted therapy, achieving an overall response rate (ORR) of $0.9 \%$. The MOSCATO trial (6) was a single-arm, prospective trial of high-throughput genomics-based targeted therapy in patients with advanced cancers, reporting an ORR of $11 \%$. In $33 \%$ of the patients (63/193), the PFS was at least 30\% longer compared with previous-line therapy (PFS2/PFS1 ratio $>1.3$ ). This ratio is an emerging treatment endpoint in PCM (7), which calculates an intra-patient PFS ratio, by dividing the PFS interval associated with molecularly guided therapy (PFS2) by the PFS interval associated with the last prior systemic therapy (PFS1). The WINTHER trial (8) demonstrated a PFS2/PFS1 ratio of $>1.5$ in $22.4 \%$ of the patients. These modest outcomes may be attributed to intra-tumour heterogeneity, clonal evolution and emergence of resistance mechanisms under treatment with targeted agents $(9,10)$. One important challenge that has been highlighted is to provide meaningful measures of outcome in small-n basket/umbrella trials. Innovative endpoints, like the PFS2/PFS1 ratio, used in the WINTHER and MOSCATO trials show promise in addressing this issue (7). These aforementioned trials represent 'unguided', broad application of CGP in solid tumours. PCM has however revolutionised treatment in specific tumour entities, including non-small cell lung cancer (NSCLC). Furthermore, in other entities, where molecularly guided treatment is not currently international standard, such as pancreatic cancer, performing CGP with NGS achieved a relevant increase in overall survival among patients in whom an actionable molecular alteration was identified in a retrospective register analysis [2.58 years (95\% CI: 2.39 to not reached) vs. 1.51 years (95\% CI: $1.33-1.87)$; $\mathrm{P}=0.0004]$ (11). Within the MOSCATO trial itself, in the subgroup of patients with advanced biliary tract cancer, treatment with molecularly guided therapy was associated with a lower risk for death $(\mathrm{HR}=0.29 ; 95 \% \mathrm{CI}$ : 0.11-0.76; $\mathrm{P}=0.008)$, with an ORR of $33 \%$ (vs. $11 \%$ in all tumour entities in the study) and a PFS2/PFS1 ratio of $>1.3$ in $50 \%$ of the patients (vs. $30 \%$ in all tumour entities in the study) (12). Therefore, it is essential to correctly identify the tumour entities/clinical settings where PCM can lead to clinically meaningful improvements in outcome.

\section{Costs}

The sequencing of the first human genome in 2003 has been estimated to be between $\$ 500$ million and $\$ 1$ billion (13). The ongoing technological advancement in NGS techniques has led to a cost decrease of at least 5 orders of magnitude, and was projected in 2016 to lay $<\$ 1,000$ per genome in 2020 (13). A meta-analysis of trials conducted between 2005 and 2016 by Schwarze et al (14) showed that cost estimates ranged from $\$ 555$ to $\$ 5,169$ for whole exome sequencing (WES) and from $\$ 1,906$ to $\$ 24,810$ for whole genome sequencing (WGS). In one of the most recent analyses in 2016, Van Nimwegen et al (15) calculated a per-sample cost of $€ 1,669$ for WGS, $€ 792$ for WES and $€ 333$ for targeted gene panels.

However, the financial burden of PCM is currently primarily driven by the cost of targeted treatment itself, rather than by diagnostic measures. Pagès et al (16) calculated the CGP cost per patient within the MOSCATO trial in France at $€ 2,396$, which was found to be only $6 \%$ of the total treatment costs; the cost of targeted therapy per patient was $€ 31,269$. Characteristically, anticancer drugs (54\%) and hospitalizations $(35 \%)$ primarily accounted for the financial burden. For patients treated with chemotherapy, treatment costs were only slightly lower at $€ 29,183$, driven primarily by higher hospitalization costs $(+27 \%)$, whereas targeted therapy was mostly administered in the ambulatory setting. In conclusion, while the costs of genetic testing are continuously declining, the costs of targeted therapy remain significant and are not expected to decrease. On the other hand, CGP not only enables prediction of response to treatment, but also resistance, thus preventing further application of unnecessary (and costly) therapies (17). Lastly, 'unguided' treatment may also be equally financially burdensome due to the increased need for hospital admissions.

\section{Cost-effectiveness}

In a recent systematic literature review by Schwarze et al (14) in 2018, which discussed the role of WES and WGS in genetic diseases, cancer and infectious pathogens, the authors concluded that available evidence is currently insufficient to draw a definitive conclusion on whether PCM is cost-effective. When estimating cost-effectiveness in broad NGS panels (targeted panels, WES or WGS) in the context of PCM, one is faced with specific challenges. The first concerns evaluating the efficacy of PCM itself. The established measure of efficacy in the era of evidence-based medicine, the placebo-controlled phase III randomised trial, is nearly impossible to apply in the context of PCM. The number of patients included in various umbrella or basket trials in PCM is insufficient to reach significant levels of statistical power for traditional endpoints, such as overall survival or PFS. Thus, Moscow et al (2) suggested that '....the clinical implementation of PCM might involve a trade-off between a different standard of evidence for the adoption of new therapies, as is the case in patients with orphan diseases, in exchange for higher levels of precision in the assignment of treatment..... New clinical trial designs and endpoints, like the PFS2/PFS1 ration in the MOSCATO and WINTHER trials $(6,8)$, are required to better quantify treatment benefit. Furthermore, there may be additional challenges 
when addressing the cost-effectiveness of PCM, as identified by Phillips et al (18). One such challenge is the difficulty of setting an appropriate comparator for NGS, i.e., single vs. multiple gene testing vs. no genetic testing. A single NGS panel could be used cost-effectively instead of multiple analyses of single genes, for example in NSCLC (19). Moreover, broad NGS panels often result in multiple secondary findings, or variants of unknown significance. Tracking the various cost and outcome trajectories that derive from the clinical significance each of these accidentally identified variants is almost impossible in a cost-effectiveness analysis. For example, accidental identification of a germline BRCA $1 / 2$ mutation could lead to risk-reducing mastectomy, initially causing an increase in healthcare costs. However, over time, costs probably would be reduced due to decreased breast cancer incidence (20). This also highlights the challenge of defining the time frame in which costs and outcomes apply. Certain costs, such as data storage or the need for additional tests (germline or family members), are difficult to depict when accompanied by findings of unclear significance. On the other hand, secondary germline data acquired with NGS, including genetic polymorphisms predicting altered pharmacokinetics, may become relevant in future medical situations, and the potential benefits of their knowledge cannot be adequately depicted at the time of NGS testing (18).

\section{Clinical utility frameworks}

Based on accumulated experience with PCM in the research setting across various cancer types and the currently available arsenal of targeted agents, different types of tumours in which the use of NGS is deemed to be clinically meaningful in daily practice can be identified. Being able to appropriately identify the clinical settings in which PCM is clinically meaningful may be the cornerstone in improving its cost-effectiveness. The ESMO Precision Medicine Working Group recently published a guideline (21) recommending NGS testing in NSCLC, cholangiocarcinoma, prostate and ovarian cancers. While the first critical step is to identify patients who would benefit from NGS testing, the next step is to determine which of the identified variants provide the most appropriate target at the given situation, because, as discussed above, targeted treatment itself is also a primary cost driver. While this obviously requires a great degree of experience and depends on the individual situation, the actionability of any individual alteration itself can be quantified to a certain extent. The ESMO Scale for Clinical Actionability of Molecular Targets (ESCAT) (22) classifies genomic alterations based on the quality of existing evidence supporting their clinical actionability. During clinical decision making, selecting the right patient for broad NGS diagnostics and focusing on variants with better evidence for clinical utility should tip the scale further toward higher cost-effectiveness of PCM.

\section{Conclusions}

Further advancements in clinical trial design, evaluation of clinical outcomes and, most importantly, the costs associated with PCM are required for adequate assessment of its cost-effectiveness. To the best of our understanding, as targeted treatment costs are unlikely to decrease, it is the declining costs of NGS that may enable broader application of the PCM approach in the future. Additionally, internationally recognised clinical frameworks can aid with appropriate selection of the tumour entity, the clinical setting and the alteration to be targeted. Thus, the economic sustainability and cost-effectiveness of PCM can be ensured and its use in daily clinical practice can be broadened in the near future.

\section{Acknowledgements}

Not applicable.

\section{Funding}

No funding was received.

\section{Availability of data and materials}

Not applicable.

\section{Authors' contributions}

Conceptualization and methodology: $\mathrm{KC}$; investigation: $\mathrm{KC}$, JTB, LT, MA, MB and DKM; writing - original draft: KC, JTB, LT, MA, MB and DKM; writing - review and editing: KC, JTB, LT, MA, SS, MB and DKM; visualization: MB; supervision: SS; project administration: DKM. All the authors have read and approved the final manuscript.

\section{Ethics approval and consent to participate}

Not applicable.

\section{Patient consent for publication}

Not applicable.

\section{Competing interests}

The authors declare that they have no competing interests.

\section{References}

1. Jameson JL and Longo DL: Precision medicine-personalized, problematic, and promising. N Engl J Med 372: 2229-2234, 2015.

2. Moscow JA, Fojo T and Schilsky RL: The evidence framework for precision cancer medicine. Nat Rev Clin Oncol 15: 183-192, 2018.

3. Tannock IF and Hickman JA: Limits to personalized cancer medicine. N Engl J Med 375: 1289-1294, 2016.

4. Le Tourneau C, Delord JP, Gonçalves A, Gavoille C, Dubot $\mathrm{C}$, Isambert $\mathrm{N}$, Campone $\mathrm{M}$, Trédan $\mathrm{O}$, Massiani MA, Mauborgne C, et al: Molecularly targeted therapy based on tumour molecular profiling versus conventional therapy for advanced cancer (SHIVA): A multicentre, open-label, proof-of-concept, randomised, controlled phase 2 trial. Lancet Oncol 16: 1324-1334, 2015.

5. Trédan O, Wang Q, Pissaloux D, Cassier P, de la Fouchardière A, Fayette J, Desseigne F, Ray-Coquard I, de la Fouchardière C, Frappaz D, et al: Molecular screening program to select molecular-based recommended therapies for metastatic cancer patients: Analysis from the ProfiLER trial. Ann Oncol 30: 757-765, 2019. 
6. Massard C, Michiels S, Ferté C, Deley MC, Lacroix L, Hollebecque A, Verlingue L, Ileana E, Rosellini S, Ammari S, et al: High-throughput genomics and clinical outcome in hard-to-treat advanced cancers: Results of the MOSCATO 01 trial. Cancer Discov 7: 586-595, 2017.

7. Mock A, Heilig CE, Kreutzfeldt S, Huebschmann D, Heining C, Schröck E, Brors B, Stenzinger A, Jäger D, Schlenk R, et al: Community-driven development of a modified progression-free survival ratio for precision oncology. ESMO Open 4: 583, 2019.

8. Rodon J, Soria JC, Berger R, Miller WH, Rubin E, Kugel A, Tsimberidou A, Saintigny P, Ackerstein A, Braña I, et al: Genomic and transcriptomic profiling expands precision cancer medicine: The WINTHER trial. Nat Med 25: 751-758, 2019.

9. Huang M, Shen A, Ding J and Geng M: Molecularly targeted cancer therapy: Some lessons from the past decade. Trends Pharmacol Sci 35: 41-50, 2014.

10. Schmitt MW, Loeb LA and Salk JJ: The influence of subclonal resistance mutations on targeted cancer therapy. Nat Rev Clin Oncol 13: 335-347, 2016.

11. Pishvaian MJ, Blais EM, Brody JR, Lyons E, DeArbeloa P Hendifar A, Mikhail S, Chung V, Sahai V, Sohal DP, et al: Overall survival in patients with pancreatic cancer receiving matched therapies following molecular profiling: A retrospective analysis of the know your tumor registry trial. Lancet Oncol 21: 508-518, 2020

12. Verlingue L, Malka D, Allorant A, Massard C, Ferté C, Lacroix L, Rouleau E, Auger N, Ngo M, Nicotra C, et al: Precision medicine for patients with advanced biliary tract cancers: An effective strategy within the prospective MOSCATO-01 trial. Eur J Cancer 87: 122-130, 2017.

13. National Human Genome Research Institute (NHGRI): The cost of sequencing a human genome. https:/www.genome.gov/aboutgenomics/fact-sheets/Sequencing-Human-Genome-cost. Accessed December 6, 2020

14. Schwarze K, Buchanan J, Taylor JC and Wordsworth S: Are whole-exome and whole-genome sequencing approaches cost-effective? A systematic review of the literature. Genet Med 20: 1122-1130, 2018

15. Van Nimwegen KJM, Van Soest RA, Veltman JA, Nelen MR, Van Der Wilt GJ, Vissers LELM and Grutters JPC: Is the 1000 genome as near as we think? A cost analysis of next-generation sequencing. Clin Chem 62: 1458-1464, 2016.

16. Pagès A, Foulon S, Zou Z, Lacroix L, Lemare F, de Baère T, Massard C, Soria JC and Bonastre J: The cost of molecular-guided therapy in oncology: A prospective cost study alongside the MOSCATO trial. Genet Med 19: 683-690, 2017.
17. Morganti S, Tarantino P,Ferraro E,D'Amico P, Viale G, Trapani D, Duso BA and Curigliano G: Role of next-generation sequencing technologies in personalized medicine. In: P5 eHealth: An Agenda for the Health Technologies of the Future. Pravettoni G and Triberti S (eds). Springer International Publishing, Cham, pp125-154, 2020.

18. Phillips KA, Deverka PA, Marshall DA, Wordsworth S, Regier DA, Christensen KD and Buchanan J: Methodological issues in assessing the economic value of next-generation sequencing tests: Many challenges and not enough solutions. Value Health 21: 1033-1042, 2018.

19. Tan AC, Lai GGY, Tan GS, Poon SY, Doble B, Lim TH, Aung ZW, Takano A, Tan WL, Ang MK, et al: Utility of incorporating next-generation sequencing (NGS) in an Asian non-small cell lung cancer (NSCLC) population: Incremental yield of actionable alterations and cost-effectiveness analysis. Lung Cancer 139: 207-215, 2020.

20. Heemskerk-Gerritsen BAM, Menke-Pluijmers MBE, Jager A, Tilanus-Linthorst MM, Koppert LB, Obdeijn IM, van Deurzen $\mathrm{CH}$, Collée JM, Seynaeve C and Hooning MJ: Substantial breast cancer risk reduction and potential survival benefit after bilateral mastectomy when compared with surveillance in healthy BRCA1 and BRCA2 mutation carriers: A prospective analysis. Ann Oncol 24: 2029-2035, 2013.

21. MoseleF,Remon J,Mateo J,Westphalen CB,BarlesiF,LolkemaMP, Normanno N, Scarpa A, Robson M, Meric-Bernstam F, et al: Recommendations for the use of next-generation sequencing (NGS) for patients with metastatic cancers: A report from the ESMO precision medicine working group. Ann Oncol 31: 1491-1505, 2020.

22. MateoJ,Chakravarty D,Dienstmann R,Jezdic S, Gonzalez-Perez A, Lopez-Bigas N, Ng CK, Bedard PL, Tortora G, Douillard JY, et al: A framework to rank genomic alterations as targets for cancer precision medicine: The ESMO scale for clinical actionability of molecular targets (ESCAT). Ann Oncol 29: 1895-1902, 2018. 